Boundary layers in a dilute particle suspension

Foster, M.R. and Duck, P.W. and Hewitt, R.E. 2006

MIMS EPrint: 2012.19

Manchester Institute for Mathematical Sciences

School of Mathematics

The University of Manchester

\footnotetext{
Reports available from: http://eprints.maths.manchester.ac.uk/

And by contacting: The MIMS Secretary

School of Mathematics

The University of Manchester

Manchester, M13 9PL, UK
} 


\title{
PROCEEDINGS
}

\section{Boundary layers in a dilute particle suspension}

\author{
By M. R. Foster ${ }^{1}$, P. W. Duck ${ }^{2}{ }^{*}$ and R. E. Hewitt ${ }^{2}$ \\ ${ }^{1}$ Department of Aerospace Engineering, The Ohio State University, \\ Columbus, $\mathrm{OH} 43210$, USA \\ ${ }^{2}$ School of Mathematics, University of Manchester, Oxford Road, \\ Manchester M13 9PL, UK
}

The general problem of a boundary-layer flow carrying a dilute, mono-disperse suspension of small particles (together with gravitational effects) is considered. The problem is modelled using the 'dusty-gas' equations, which are a coupled equation set linking the fluid motion to that of the particle motion (both of which are modelled as continua). A number of qualitatively distinct potential scenarios are predicted. These include a variety of boundary-layer breakdowns, and the formation of shock transitions in the distribution of the particulate phase (together with the development of particlefree zones). Numerical results predicting these differing behaviours are confirmed through local asymptotic analyses of the governing equations. Although we consider a general class of boundary layer, our results are compared and contrasted with previous studies of specific cases, most notably the constant freestream fluid velocity case (akin to the 'clean' Blasius boundary layer). In the case of a boundary-layer flow driven by a linearly retarding free stream (the analogue of the classical 'Howarth' boundary-layer problem), the effects of the particle phase are surprisingly seen to (slightly) delay the separation of the boundary layer.

Keywords: multiphase flow; boundary layer; particle-laden

\section{Introduction}

Prediction of the dispersal of very large numbers of very small, solid particles in a continuous fluid medium (either liquid or gas) is a common and challenging problem in a wide variety of settings. Examples are found in a number of processes in the automotive, chemical and coatings industries and in environmental applications such as the disposal of wastewater-borne particulates in the coastal zone and the prediction of particulate deposition from urban atmospheric gas emissions. Accordingly, the problems associated with predicting the flow characteristics and general properties of such dilute-solution systems are not only of fundamental interest from a fluid mechanical standpoint but also of great economic, social and environmental significance. Much scientific effort has been applied to these multiphase systems, particularly in the chemical engineering, environmental fluid mechanics and applied mathematics communities.

* Author for correspondence (duck@ma.man.ac.uk). 
The role of boundary layers in dilute suspensions of small mono-disperse particles has been studied for over fifty years. Marble (1970) summarized much of the early work in this field, and Osiptsov (1997) subsequently gave a wideranging review of the more recent developments. Using what have since become commonly known as the 'dusty-gas' equations, the Blasius-like boundary-layer problem has predictably received much attention. The original studies are described in Singleton (1965) and Soo (1968), with more recent work being Osiptsov (1980), Asmolov (1995) and Wang \& Glass (1988). Some common features emerge in these studies, the most important of which are as follows. (i) near the leading edge, in the absence of any 'particle viscosity', the particles slip over the surface, while far downstream, the particles generally move with the flow. (ii) There is a singularity in the particle volume fraction that develops on the wall at a particular downstream location; solutions beyond this point are problematic. It has been suggested by some authors (including Osiptsov 1988) that the introduction of the Saffman (1965) force can mitigate the problem in the dusty analogy to the Blasius boundary layer (however, see our comments in §5). Note that Wang \& Glass (1988) continued their numerical results downstream of the singularity, an approach which is, as we shall show, not valid.

Modification of the boundary layer by buoyancy of the embedded particles has received less attention. Foster et al. (2003) showed that gravitational effects greatly modify the Kármán boundary layers that occur in connection with a spinup, showing the potential for a 'shock' to occur in the particle concentration distribution. Such shock structures have also been reported in Ungarish (1993) and Ungarish \& Greenspan (1983), for example, and are due to the kinematicwave structure of the particle-mass conservation equations.

In a recent study of a laminar wall jet, Duck et al. (2004) have found that the self-similar structure of the particle-free jet, described first by Glauert (1956), becomes non-similar when a dilute suspension of particles is considered. Furthermore, a singularity in volume fraction occurs at the wall (linked to a stagnation point in the particle motion) and is an inherent prediction of the model. In the work of Duck et al. (2004), the singularity cannot be removed in any simple manner and suggests that a non-dilute model is required in the local neighbourhood of the singularity. The direction of the gravitational force acting on the particle phase has a strong impact on the character of this singularity. The present authors also determined that, apparently unlike the Blasius-like boundary layer, the inclusion of a Saffman force, which is small along the length of the wall jet, does not remove this singularity.

In this paper, we explore the character of particle-laden boundary layers more generally. We shall formulate the problem for general freestream variations of the fluid flow, and present results for both the Falkner-Skan-type freestream variations,

$$
u^{*}=U_{\infty}\left(\frac{x^{*}}{L}\right)^{m}, \quad 0 \leq m \leq 1,
$$

and that considered by Howarth (1934),

$$
u^{*}=U_{\infty}\left[1-\frac{x^{*}}{L}\right] .
$$




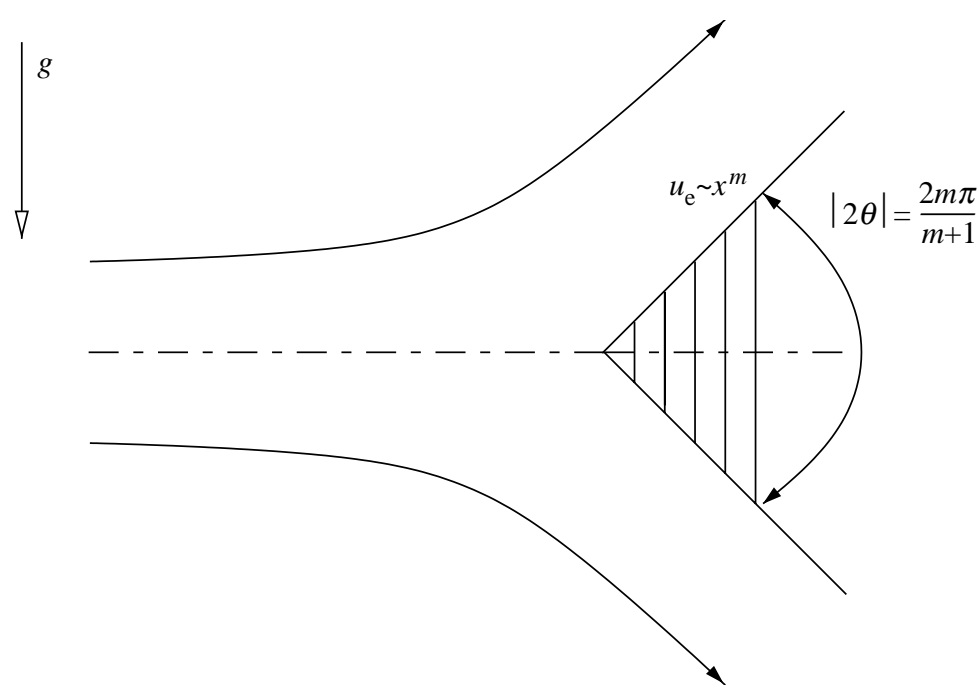

Figure 1. A flow geometry appropriate for the Falkner-Skan-type edge conditions; although the results we present for the boundary layer developing on the impermeable wedge are not restricted to having self-similarity and are more general in nature. We assume that the local gravitational forcing is aligned as shown and $\theta \in[0, \pi / 2]$; thus the upper boundary layer is such that $\mathcal{K}>0$, while the lower boundary layer has $\mathcal{K}<0$, where $\mathcal{K}$ is as defined in $(2.6)$.

It should be emphasized, however, that although we discuss Falkner-Skan-like conditions at the edge of the boundary layer, the solution of the boundary layer itself is not self-similar (the usual Falkner-Skan self-similar forms are certainly inappropriate for the general governing equations for a dusty-gas and one must consider the general spatially developing flow). Because of the potentially complex nature of the Euler flow outside the boundary layer, we are not at liberty to arbitrarily specify the edge values of either the particle velocity or the volume fraction in the external flow. We shall calculate admissible external flow conditions, and use these as appropriate boundary conditions for the boundarylayer solutions.

\section{The dusty-gas equations of motion}

The dimensional continuity equations for an incompressible fluid carrying small, solid particles are taken to be of the form

$$
\nabla^{*} \cdot \boldsymbol{u}^{*}=0 \quad \text { and } \quad \nabla^{*} \cdot\left(\alpha \boldsymbol{u}_{\mathrm{p}}^{*}\right)=0,
$$

where $\boldsymbol{u}^{*}$ is the velocity vector of the fluid component, $\boldsymbol{u}_{\mathrm{p}}^{*}$ is the particlecontinuum velocity vector, and $\alpha$ is the particle volume fraction (assumed to be small). The equations for the carrying fluid and the dilute particle phase are assumed to be steady and to take the form

$$
\begin{gathered}
\rho_{\mathrm{f}}\left(\boldsymbol{u}^{*} \cdot \nabla^{*}\right) \boldsymbol{u}^{*}+\nabla^{*} p^{*}=\mu \nabla^{* 2} \boldsymbol{u}^{*}-\boldsymbol{F}^{*}-\rho_{\mathrm{f}} \boldsymbol{g}, \\
\rho_{\mathrm{p}} \alpha\left(\boldsymbol{u}_{\mathrm{p}}^{*} \cdot \nabla^{*}\right) \boldsymbol{u}_{\mathrm{p}}^{*}=\boldsymbol{F}^{*}-\rho_{\mathrm{p}} \alpha \boldsymbol{g}+\rho_{\mathrm{f}} \alpha \boldsymbol{g},
\end{gathered}
$$


where $\mu$ is the fluid viscosity coefficient, $\left(\rho_{\mathrm{f}}, \rho_{\mathrm{p}}\right)$ are the fluid and particle densities. Note that a buoyancy term is the final term in $(2.2 b)$. We have replaced factors of $(1-\alpha)$ with 1 , in accordance with the dilute limit. The quantity $\boldsymbol{F}^{*}$ is the interaction force between the fluid and the particle continuum.

The gravitational acceleration vector can, in general, be taken at some angle to the surface; however, in order to reduce our parameter space by one dimension, we assume that gravity acts perpendicularly to the on-coming flow. This restriction leads to a connection between the angle $\theta$ and the parameter $m$ (in (1.1), as arises in wedge flows; see figure 1), namely $\theta=\pi m /(m+1)$. Clearly, this relationship is the classical one for an irrotational outer flow. However, one can assume that the inviscid fluid flow (even when particle-laden) is irrotational and then evaluate the particle velocity and volume fraction associated with that motion, but there may well be situations where the particle solution becomes singular (for example) as we shall see below. In those circumstances, the irrotational assumption may not, in fact, be proper. It should be pointed out that the basic equation set $(2.1),(2.2 a)$ and $(2.2 b)$ falls into the same class as that taken in a number of recent successful studies by other authors, including Slater \& Young (2001), Hernández (2001), Narayanan \& Lakehal (2002) and Féraille \& Casalis (2003).

Complete specification of the flow and closure of the model, requires that the fluid-particle interaction force be specified. In our case, following previous work in this area, assuming small, spherical particles (radius a), we assume Stokes drag,

$$
\boldsymbol{F}_{\text {Stokes }}^{*}=\frac{9 \mu}{2 a^{2}} \alpha\left(\boldsymbol{u}-\boldsymbol{u}_{\mathrm{p}}\right) \text {. }
$$

In addition, many studies, including Duck et al. (2004), have included the Saffman (1965) force, which can be written as

$$
\boldsymbol{F}_{\text {Saffman }}^{*}=\frac{c \alpha}{a}\left(4 \mu^{2} \rho_{\mathrm{f}}^{2} \mathbf{D}_{i j} \mathbf{D}_{i j}\right)^{1 / 4} \boldsymbol{n} \times\left(\boldsymbol{u}^{*}-\boldsymbol{u}_{\mathrm{p}}^{*}\right),
$$

where $\mathbf{D}_{i j}$ is the deformation tensor, $c$ is a numerical constant and $\boldsymbol{n}$ is a unit vector normal to the plane of the two-dimensional flow. However, the Saffman force does not appear to play a significant role in the problems discussed in this paper; we quantify this conclusion in $\S 5$.

\section{(a) The boundary-layer equations}

If the dimensional fluid speed at the edge of the boundary layer is denoted by $U_{\infty} u_{\mathrm{e}}(x)$, then the velocity vectors of the fluid and particles are $U_{\infty}\left(u, R e^{-1 / 2} v\right)$, $U_{\infty}\left(u_{\mathrm{p}}, R e^{-1 / 2} v_{\mathrm{p}}\right)$, respectively, and the corresponding dimensionless coordinates are $x, y$ normalized using a characteristic streamwise length-scale $L$ and $L R e^{-1 / 2}$, respectively, ${ }^{1}$ and then assuming the Reynolds number $R e=$ $U_{\infty} L / \nu \gg 1$ (the kinematic viscosity of the fluid being $\nu=\mu / \rho_{\mathrm{f}}$ ), the dusty-gas equations can be written,

$$
\begin{aligned}
u u_{x}+v u_{y}+\bar{p}_{x} & =u_{y y}-\beta \alpha\left(u-u_{\mathrm{p}}\right), \\
u_{\mathrm{p}} u_{\mathrm{p} x}+v_{\mathrm{p}} u_{\mathrm{p} y} & =\frac{\beta}{\gamma}\left(u-u_{\mathrm{p}}\right),
\end{aligned}
$$

\footnotetext{
${ }^{1}$ Note that, for a given value of $m$, a sensible definition can be given for $L$ in terms of the parameters of that particular problem; for $m=0, L$ is the actual length of the plate.
} 


$$
\begin{aligned}
u_{\mathrm{p}} v_{\mathrm{p} x}+v_{\mathrm{p}} v_{\mathrm{p} y} & =\frac{\beta}{\gamma}\left(v-v_{\mathrm{p}}\right)-\mathcal{K} \cos \theta \\
u_{x}+v_{y} & =0 \\
u_{\mathrm{p}} \alpha_{x}+v_{\mathrm{p}} \alpha_{y} & =-\alpha\left(u_{\mathrm{p} x}+v_{\mathrm{p} y}\right) .
\end{aligned}
$$

The dimensionless quantities that arise in this system are

$$
\beta=\frac{9}{2} \frac{\nu L}{U_{\infty} a^{2}}, \quad \gamma=\frac{\rho_{\mathrm{p}}}{\rho_{\mathrm{f}}}, \quad \mathcal{K}=\frac{g L R e^{1 / 2}}{U_{\infty}^{2}}\left(1-\frac{1}{\gamma}\right),
$$

and the usual requirements for the validity of this equation set are that $\gamma \gg 1$, $\beta \gg 1$, max $\alpha \ll 1$. The ordering of $\beta$ implies that the boundary layer is much thicker than the particle size. The requirement that the distance between particles also be much smaller than the boundary-layer width is $\beta \alpha^{2 / 3} \gg 1$. This is consistent with the analysis that follows but we note that this may be difficult to achieve in a laboratory setting. The rationale for maintaining parameters of extreme size in the governing equations is that the combinations $\beta / \gamma, \beta \alpha$ and $\mathcal{K}$ all remain order-one parameters. In the boundary-layer equations $(2.5 a)-(2.5 e), \bar{p}$ is a pressure with the gravitational potential included. The boundary conditions for the fluid phase are

$$
u=v=0 \quad \text { on } y=0, \quad u \rightarrow u_{\mathrm{e}}(x) \quad \text { as } y \rightarrow \infty .
$$

However, the choice of boundary conditions for the particle phase is somewhat more subtle (and will be discussed later), but notionally we expect that

$$
u_{\mathrm{p}} \rightarrow u_{\mathrm{pe}}(x) \quad \text { and } \quad \alpha \rightarrow \alpha_{\mathrm{e}}(x), \quad \text { for } y \rightarrow \infty .
$$

In this paper, we assume that the fluid outside the boundary layer is irrotational, leading to two types of edge velocities, given by

$$
u_{\mathrm{e}}(x)=x^{m}, \quad 0 \leq m \leq 1 \quad \text { and } \quad u_{\mathrm{e}}(x)=1-x,
$$

(consistent with (1.1) and (1.2), respectively). The first of these is due to the flow of a fluid past a wedge of half-angle $\theta$, as shown in figure 1 . Precisely, what are appropriate functional forms for $u_{\mathrm{pe}}$ and $\alpha_{\mathrm{e}}$ connected to these fluid flows is discussed in $\S 3$.

The volume fraction equation (2.5e) importantly involves the divergence of the particle fluid velocity, which in general is non-zero. Intuitively, we expect the divergence of the particle field to be of some significance since, in the absence of sources/sinks, any assumption that the external flow has a uniform volume fraction can only be consistent if the particle flow field is also divergence free. By taking the divergence of the particle momentum equation, we obtain a transport equation for the particle velocity divergence, $\boldsymbol{\nabla} \cdot \boldsymbol{u}_{\mathrm{p}} \equiv \mathcal{D}$, in the form

$$
\left(\boldsymbol{u}_{\mathrm{p}} \cdot \nabla\right) \mathcal{D}+\frac{\beta}{\gamma} \mathcal{D}=-\left(u_{\mathrm{p} x}\right)^{2}-\left(v_{\mathrm{p} y}\right)^{2}-2 u_{\mathrm{p} y} v_{\mathrm{p} x}
$$




\section{The outer flow and conditions at the boundary-layer edge}

Perhaps surprisingly, inviscid flows of dilute suspensions have received little analysis to date, and the question of an appropriate particle-phase flow field for flow past a wedge is not entirely straightforward. It can easily be shown that a scenario in which both the fluid and the dispersed phase are irrotational everywhere is not possible. Nevertheless, for streaming flow past any object, an irrotational flow seems plausible. The consequences of this assumption are that there must be variation in the volume fraction in the flow and that the particle phase is rotational. Though we do not examine further the global outer flow characteristics, evaluating the boundary-layer equations at the outer 'edge' of the boundary layer does provide conditions for the boundary layer. Using equations $(2.5 b)$, the $y$-derivative of $(2.5 c)$ and $(2.5 e)$, all evaluated at the layer edge leads to

$$
u_{\mathrm{pe}} u_{\mathrm{pe}}^{\prime}=\frac{\beta}{\gamma}\left(u_{\mathrm{e}}-u_{\mathrm{pe}}\right), \quad u_{\mathrm{pe}} E_{\mathrm{e}}^{\prime}+E_{\mathrm{e}}^{2}+\frac{\beta}{\gamma} E_{\mathrm{e}}=-\frac{\beta}{\gamma} u_{\mathrm{e}}^{\prime}, \quad u_{\mathrm{pe}} \alpha_{\mathrm{e}}^{\prime}+\alpha_{\mathrm{e}} \mathcal{D}_{\mathrm{e}}=0,
$$

where $E_{\mathrm{e}}(x)=\partial v_{\mathrm{p}} / \mathrm{\partial} y(y \rightarrow \infty), u_{\mathrm{pe}}(x)=u_{\mathrm{p}}(y \rightarrow \infty)$ and $\mathcal{D}_{\mathrm{e}}(x)=\mathcal{D}(y \rightarrow \infty)=u_{\mathrm{pe}}^{\prime}+$ $E_{\mathrm{e}}$ are the relevant functions evaluated as $y \rightarrow \infty$ and primes denote differentiation with respect to the streamwise coordinate $x$. To arrive at this system, we have assumed that $\partial u_{\mathrm{p}} / \partial y, \partial^{2} v_{\mathrm{p}} / \partial y^{2}$ vanish for $y \rightarrow \infty$. We also retain the transport equation for $\mathcal{D},(2.10)$, which when evaluated at the layer edge, becomes

$$
u_{\mathrm{pe}} \mathcal{D}_{\mathrm{e}}^{\prime}+\frac{\beta}{\gamma} \mathcal{D}_{\mathrm{e}}+\left(u_{\mathrm{pe}}^{\prime}\right)^{2}+E_{\mathrm{e}}^{2}=0 .
$$

From (3.1) and (3.2), it follows that

$$
\begin{gathered}
u_{\mathrm{pe}}\left[u_{\mathrm{pe}}^{\prime}+\frac{\beta}{\gamma}\right]=\frac{\beta}{\gamma} u_{\mathrm{e}}, \\
\left(u_{\mathrm{pe}} \frac{\mathrm{d}}{\mathrm{d} x}+\frac{\beta}{\gamma}\right)\left(u_{\mathrm{pe}} \frac{\alpha_{\mathrm{e}}^{\prime}}{\alpha_{\mathrm{e}}}\right)=\left(u_{\mathrm{pe}}^{\prime}\right)^{2}+E_{\mathrm{e}}^{2} .
\end{gathered}
$$

Thus, for a given fluid edge behaviour $u_{\mathrm{e}}(x),(3.3 a)$ can be solved for the streamwise particle motion $u_{\mathrm{pe}}(x)$, then the second of (3.1) for the particle motion normal to the boundary $E_{\mathrm{e}}$, and finally $(3.3 b)$ determines the external volume fraction $\alpha_{\mathrm{e}}(x)$.

Below, we focus our attention on freestream fluid velocities of the form $u_{\mathrm{e}}(x)=x^{m}$, then later in the paper, we will go on to broaden the class of freestream variations by considering Howarth-type freestreams (corresponding to $(1.2))$.

\section{(a) $m=0$ : a Blasius-type external flow}

We first consider the Blasius-like case, for which $m=0$. There is a trivial exact solution to $(3.3 a), u_{\mathrm{pe}}=1$, together with $\alpha_{\mathrm{e}}=\alpha_{\mathrm{e}}(x=0)=\alpha_{0}$ (constant) and $E_{\mathrm{e}} \equiv 0$ from $(3.3 b)$. Since the external particle flow is divergence free, we can sustain a spatially uniform external volume fraction. 


\section{(b) $m=1$ : Hiemenz flow}

For the Falkner-Skan profiles with $m=1$, the general solution of $(3.3 a)$ can easily be found to be

$$
\left(u_{\mathrm{pe}}-r_{1} x\right)^{r_{1}}\left(u_{\mathrm{pe}}+r_{2} x\right)^{r_{2}}=C, \quad C=\text { const. },
$$

where

$$
r_{1,2}=\mp \frac{\beta}{2 \gamma}+\sqrt{\frac{\beta^{2}}{4 \gamma^{2}}+\frac{\beta}{\gamma}} .
$$

The corresponding solution for $E_{\mathrm{e}}$ may also be found, namely,

$$
\frac{E_{\mathrm{e}}+s_{1}}{E_{\mathrm{e}}+s_{2}}=\bar{C} x^{-\left(s_{2}-s_{1}\right) / r_{1}},
$$

where $\bar{C}$ is another constant and

$$
s_{1,2}=\frac{\beta}{2 \gamma} \mp \sqrt{\frac{\beta^{2}}{4 \gamma^{2}}-\frac{\beta}{\gamma}} .
$$

Further, the equation for $\alpha_{\mathrm{e}}$ may be integrated, and the solution that is bounded at the origin is given by

$$
\alpha_{\mathrm{e}}=C^{\prime} x^{\gamma\left(r_{1}^{2}+s_{1}^{2}\right) / \beta r_{1}},
$$

with the constant $C^{\prime}$ not specified. There are two possibilities: (i) if $C=0$, then $u_{\mathrm{pe}}=r_{1} x$, corresponding to a Hiemenz flow; this appears to be a self-similar case, but it turns out that the only such solution is one which is entirely particle free, i.e. $\alpha \equiv 0$ inside the boundary layer, and so we do not investigate this case further; (ii) if $C=U_{\mathrm{p} 0}^{r_{1}+r_{2}}$, then $u_{\mathrm{pe}} \sim U_{\mathrm{p} 0}$, a constant, at $x=0$, so this case falls into the same class as those discussed below.

\section{(c) $1>m>0$ : wedge flows}

There are again two possibilities: (i) $u_{\mathrm{pe}}(0)=0$, and (ii) $u_{\mathrm{pe}}(0)=U_{\mathrm{p} 0}$ (const.). Thus, these two scenarios, noted above for $m=1$, appear to exist for all $m$. In this paper, for wedge flows, we examine just case (ii) flows.

The small- $x$ behaviour is found from (3.1) to take the form

$$
u_{\mathrm{pe}} \sim U_{\mathrm{p} 0}-\frac{\beta}{\gamma} x+\frac{\beta}{\gamma U_{\mathrm{p} 0}} \frac{x^{m+1}}{m+1}+\cdots .
$$

The quantity $U_{\mathrm{p} 0}$ is an arbitrary constant, which although retained explicitly in the analysis as a parameter, will be set to unity in the numerical calculations; we shall comment further on the appearance of this arbitrary constant in $\S 8$. We turn now to expressions for the volume fraction. Asymptotic solutions for $E_{\mathrm{e}}$ for small $x$ may be found, and then the solution to $(3.3 b)$ is

$$
\alpha_{\mathrm{e}}=\alpha_{0}\left(1+\frac{\beta x}{\gamma U_{\mathrm{p} 0}}+\cdots\right) .
$$



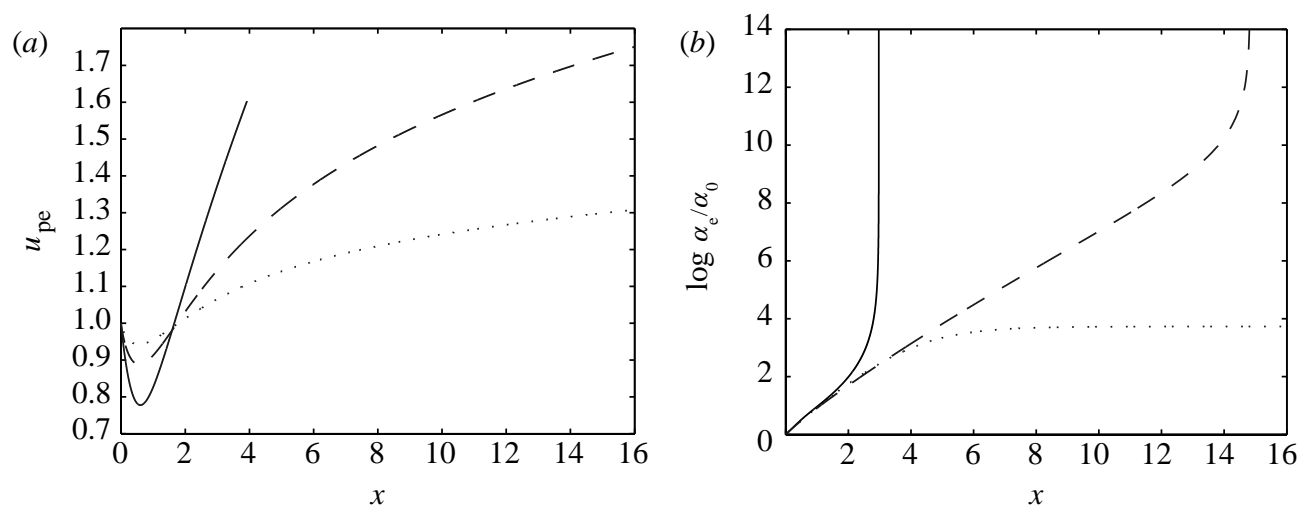

Figure 2. Development of the edge quantities $(a) u_{\mathrm{pe}}$ and $(b) \alpha_{\mathrm{e}}$; solid, $m=0.50$; dashed, $m=0.211$; dotted, $m=0.10 ;$ all with $\beta / \gamma=1$.

Figure $2 a$ shows the downstream development of $u_{\mathrm{pe}}$ at three selected values of $m$, namely $0.1,0.211$ and 0.5 , with $\beta / \gamma=1$, as determined from the full numerical solution of (3.1). Although, this figure indicates a relatively benign behaviour, inspection of the corresponding results for $\alpha_{\mathrm{e}}$, as shown in figure $2 b$, reveal a singular response at the two larger values of $m$. Note that for the outer-edge problem, the results will depend linearly on $\alpha_{0}$ and this scaling has, therefore, been applied to the presented data.

The nonlinear equation for $\alpha_{\mathrm{e}}$ (and also $E_{\mathrm{e}}$ ) often develops a singularity at a finite value of $x$. In fact, it is straightforward to show that near this location $x_{\mathrm{s}}$, the approximate solution is

$$
E_{\mathrm{e}} \sim \frac{u_{\mathrm{pe}}\left(x_{\mathrm{s}}\right)}{x-x_{\mathrm{s}}}, \quad \alpha_{\mathrm{e}} \sim \frac{1}{x-x_{\mathrm{s}}}
$$

Numerical solution verifies this simple singularity structure, and also indicates that this outer-flow singularity develops only in a restricted range for $m$, namely, for $m>m_{\mathrm{s}}$, where we have determined $m_{\mathrm{s}}$ to be 0.2109 (approximately).

In the cases when solutions do extend (infinitely) far downstream, it is straightforward to show that (see also appendix A)

$$
u_{\mathrm{pe}} \sim x^{m}-\frac{m \gamma}{\beta} x^{2 m-1}+\cdots
$$

and so to leading order the freestream particles have the same velocity as that of the fluid, and

$$
\alpha_{\mathrm{e}} \sim c_{1}\left(1-\frac{2 m^{2} \beta}{\gamma(1-m)} x^{m-1}+\cdots\right)
$$

where $c_{1}$ is a constant (determined through integration downstream from the leading edge). 


\section{(d) Howarth flow}

A final case examined in this paper is the irrotational outer flow $u_{\mathrm{e}}=1-x$; an example due to Howarth (1934). In this case, equation (3.3a) leads to the implicit solution

$$
\left(\frac{u_{\mathrm{pe}}-s_{1}(1-x)}{U_{\mathrm{p} 0}-s_{1}}\right)^{s_{1}}=\left(\frac{u_{\mathrm{pe}}-s_{2}(1-x)}{U_{\mathrm{p} 0}-s_{2}}\right)^{s_{2}},
$$

and the exact solution for $E_{\mathrm{e}}$ that is bounded at $x=1$ is $E_{\mathrm{e}}=-r_{2}$. The result for $\alpha_{\mathrm{e}}$ cannot be given in any simple closed form, although the small- $x$ behaviour is given by

$$
u_{\mathrm{pe}} \sim U_{\mathrm{p} 0}+\frac{\beta}{\gamma}\left(\frac{1}{U_{\mathrm{p} 0}}-1\right) x+\cdots, \quad \alpha_{\mathrm{e}} \sim \alpha_{0}\left(1+\frac{r_{2}^{2} \gamma}{\beta} x+\cdots\right),
$$

where $r_{1,2}, s_{1,2}$ are given by $(3.4 b)$ and $(3.5 b)$, respectively.

\section{Boundary-layer results for $0 \leq m<1$ for $\mathcal{K} \geq 0$}

When there are no gravitational effects $(\mathcal{K}=0)$, then for $m \geq 0$ the dispersed phase velocity vector is directed towards the wall. This can be confirmed by a local analysis close to the leading edge (i.e. small $x$ ), and is also confirmed in our numerical computations. This is in spite of the bidirectional nature of the transverse fluid velocity component $(v)$ in cases for which $m>0$.

Positive values of the buoyancy parameter $\mathcal{K}$ reinforce this downward migration of the particle suspension. Consequently, the hyperbolic nature of the equations for the particle velocity vector implies that the component of particle velocity normal to the wall, $v_{\mathrm{p}}$, cannot be specified at the wall, rather conditions at the outer edge of the boundary layer for the particle phase must be specified. This was also the case with the wall jet analysed by Duck et al. (2004).

However, when $\mathcal{K}=0$ with the specified conditions at the leading edge $\left(u_{\mathrm{p}}=U_{\mathrm{p} 0}, v_{\mathrm{p}}=0\right)$, it is easy to see (from evaluating $(2.5 b)$ and $(2.5 c)$ on $\left.y=0\right)$ that the particle behaviour on the wall is

$$
v_{\mathrm{p}}(y=0)=0, \quad u_{\mathrm{p}}(y=0)=U_{\mathrm{p} 0}-\frac{\beta}{\gamma} x .
$$

Although, these conditions were not imposed on the system, they arose naturally during the course of the computations. This certainly indicates the prospect of a singularity at the point $x=\gamma U_{\mathrm{p} 0} / \beta$, even without any computation being performed. This is rather unusual in the context of boundary layers (including the analogous wall jet problem of Duck et al. 2004), where generally singularity location is very much dependent on the details of the boundary-layer solution (and is the situation in the present study when $\mathcal{K}>0$ ). This difference arises because the wall is itself a characteristic when $\mathcal{K}=0$ and, therefore, the solution at $y=0$ is unaffected by the behaviour above it, being determined instead by the flow at the leading edge only.

Naively approaching the case $\mathcal{K}<0$ with precisely the same numerical method used for $\mathcal{K} \geq 0$ leads to spurious computational results which are highly sensitive 
to grid choices immediately downstream of the leading edge. This regime is fundamentally different, because of the bidirectional nature of $v_{\mathrm{p}}$ when $\mathcal{K}<0$, which can result in shock-like transitions in the particle volume distribution. Given this distinction, we shall postpone discussion of this case until $\S 6$.

\section{(a) The numerical formulation}

Duck et al. (2004) have found that the presence of a dispersed phase in the flow destroys the self-similar character found in the 'clean' case. One can write, from the outset, the boundary-layer equations in Falkner-Skan form, with $u_{\mathrm{e}} \sim x^{m}$ and a boundary-layer thickness scaling as $x^{(1-m) / 2}$, but this leads to contradictions, so that no self-similar structure is possible for $0 \leq m<1$. Although, the $m=1$ case looks to be a good candidate for a fully self-similar solution, similarity requirements imply that $\alpha$ can be a function of $y$ only, and compatibility with (3.6) implies that the only solution that is self-similar is one in which there are no particles, i.e. $\alpha \equiv 0$ (as mentioned in $\S 3$ ).

Nonetheless, even for general $m$, for small $x$, the boundary layer begins to develop according to the $\alpha=0$ Falkner-Skan similarity form, and so it is convenient to use these variables numerically to capture the initial stages accurately. It can be shown that the following forms are consistent with the small $x$ behaviour of the flow and particle equations:

$$
\begin{aligned}
& u=x^{m} U(\xi, \eta), \quad v=x^{(m-1) / 2} V(\xi, \eta), \quad u_{\mathrm{p}}=U_{\mathrm{p} 0}+x U_{\mathrm{p}}(\xi, \eta), \\
& v_{\mathrm{p}}=x^{(m+1) / 2} V_{\mathrm{p}}(\xi, \eta), \quad \alpha=\alpha_{0}+x \bar{\alpha}(\xi, \eta), \quad \xi=x^{(1-m) / 2} .
\end{aligned}
$$

The similarity variable is of the Falkner-Skan type, namely $\eta=y x^{(m-1) / 2}$. Rather than assuming the pure-similarity form for the streamwise fluid velocity in the freestream (i.e. $U(\xi, \eta \rightarrow \infty) \rightarrow 1$ ), in the interests of generality we shall instead assume the form

$$
U(\xi, \eta \rightarrow \infty) \rightarrow U_{\mathrm{e}}(\xi)
$$

although we will take $U_{\mathrm{e}}(0)=1$ in order to connect to the leading-edge similarity structure.

The equations governing the transformed quantities (4.2) are easily obtained by substitution, and are solved subject to the edge conditions. ${ }^{2}$ The quantities $U_{\text {pe }}$ and $\bar{\alpha}_{\mathrm{e}}$ (that is, the quantities $U_{\mathrm{p}}$ and $\bar{\alpha}$ evaluated at the edge of the boundary layer, i.e. as $\eta \rightarrow \infty)$ must be determined numerically in conjunction with the rest of the computation. To achieve this, we suppose that as $\eta \rightarrow \infty$, $V_{\mathrm{p}} \rightarrow \bar{E}_{\mathrm{e}} \eta+V_{\mathrm{pe}}$, consistent with $V \rightarrow-m U_{\mathrm{e}} \eta+V_{\mathrm{e}}$ in this limit. The resulting edge quantities are then determined by the transformed versions of (3.1), (3.3a) and $(3.3 b)$ in a manner similar to that of $\S 3$.

${ }^{2}$ We note that some slight simplification is achieved via the transformation

$$
U_{\mathrm{p}} \rightarrow \frac{\beta}{\gamma} U_{\mathrm{p}}, \quad V_{\mathrm{p}} \rightarrow\left(\frac{\beta}{\gamma}\right)^{1 /(1-m)} V_{\mathrm{p}}, \quad \xi \rightarrow\left(\frac{\gamma}{\beta}\right)^{1 / 2} \xi, \quad \alpha \rightarrow\left(\frac{\beta}{\gamma}\right)^{1 /(1-m)} \alpha, \quad U_{\mathrm{p} 0} \rightarrow\left(\frac{\gamma}{\beta}\right)^{m /(1-m)} U_{\mathrm{p} 0},
$$

with no rescaling for $(U, V)$. However, in this paper we prefer to minimize the notational changes that would result from such a transformation and maintain the more physically relevant scales. 

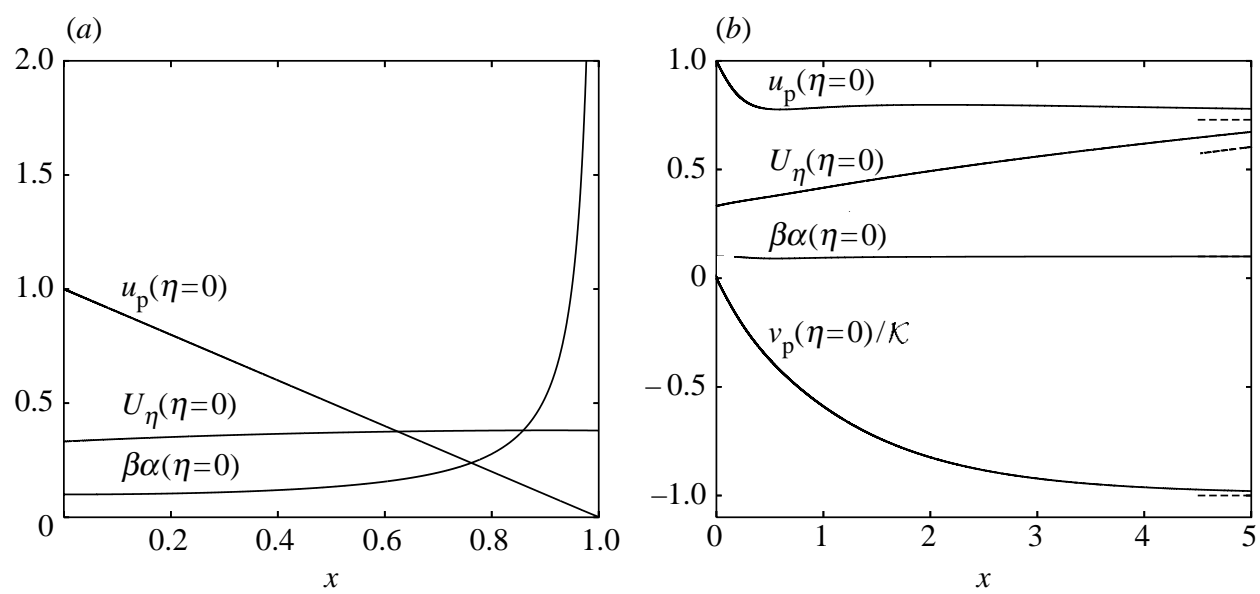

Figure 3. The development of wall values with $x$ for $m=0, \beta / \gamma=1$. (a) $\mathcal{K}=0$. In case $(b)$, the leading-order asymptotic forms for $x \rightarrow \infty$ are shown for $x>4.5$; the asymptotic form for $\beta \alpha$ is indistinguishable from the data presented $(\mathcal{K}=10)$.

A key point is the choice of boundary conditions on the wall, $\eta=0$. For the fluid, this is straightforward and standard, merely the impermeability and no-slip conditions,

$$
U(\eta=0)=V(\eta=0)=0 .
$$

The particle phase is crucially different and, as discussed above, the hyperbolic nature of the particle phase necessitates the imposition of edge $(\eta \rightarrow \infty)$ conditions on $U_{\mathrm{p}}, V_{\mathrm{p}}$ and $\alpha$.

A standard Crank-Nicolson procedure (marching in $\xi$ ) is adopted, with second-order finite differencing in $\eta$ coupled with Newton iteration.

\section{(b) The numerical results}

The first set of boundary-layer results is presented in figure $3 a$ for the case $m=0, \mathcal{K}=0, \beta \alpha_{0}=0.1, \beta / \gamma=1$. (We recall here that the combinations $\beta \alpha_{0}$ and $\beta / \gamma$ are $\mathcal{O}(1)$ quantities in the dusty-gas model.) The immediate observation is the apparent 'blow-up' of the particle concentration as the location $x=1$ is approached. It must be emphasized (again) that as the particle concentration becomes large, the basic underlying assumption of the dusty-gas model becomes invalid locally. A second, significant observation is the apparent linear downstream decrease of $u_{\mathrm{p}}(y=0)$ towards zero as $x \rightarrow 1$ (consistent with (4.1) above). An additional third point (not illustrated in the figure) is that $v_{\mathrm{p}}(y=0)=0$ for all $x$. The last two features can be readily confirmed from the equations of motion (specifically $(2.5 d)$ and $(2.5 c)$ ) evaluated on $y=0$, while all three features are part of the analysis of the $\alpha$ singularity, studied in $\$ 5$. We note that the response of the fluid (as measured by the wall shear stress, $U_{\eta}(\eta=0)$ ) is relatively benign. This apparent singularity in the dilute model is to be expected, given the associated stagnation point of the particle continuum encountered on the wall at this point. 
(a)

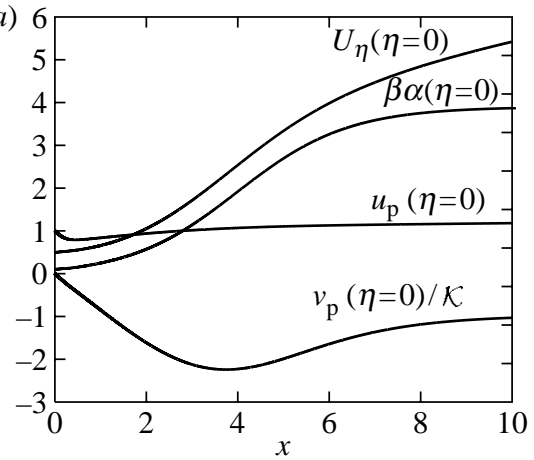

(b)

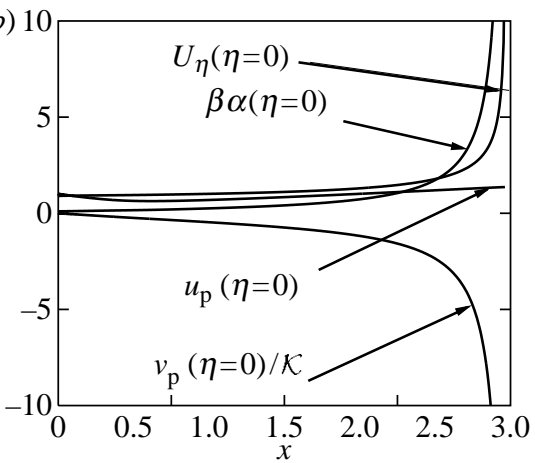

Figure 4. The development of wall values with $x$ for $\mathcal{K}=10, \beta / \gamma=1$. (a) $m=0.1$ and $(b) m=0.5$.

Figure $3 b$ shows the wall values when $m=0$ with a buoyancy parameter $\mathcal{K}=10$ (all other parameters correspond to those taken in figure $3 a$ ). The effect of buoyancy is clearly seen to be drastic, with the motion continuing unabated downstream, with no hint of any breakdowns in any of the flow or particle quantities. The asymptotic structure of the far downstream flow is given in appendix A, and this behaviour is indicated on figure $3 b$.

We now turn to consider cases for which $m \neq 0$. Computations in which $m=0.1, \mathcal{K}=0, \beta \alpha_{0}=0.1, \beta / \gamma=1$ show little qualitative difference from those presented in figure $3 a$. The same features occur, with $v_{\mathrm{p}}(y=0)=0$, for $0 \leq x<1$, $x=1$ is a stagnation point for the particle motion, accompanied by an apparent singular response in the volume fraction, $\alpha$. A computation that includes the effects of buoyancy is highlighted by figure $4 a$. In this case, $m=0.1$ and all other parameters are the same as those chosen for figure $3 b$. The results show some features in line with the $m=0, \mathcal{K}=10$ results (figure $3 b$ ), with the solution extending far downstream.

Figure $4 b$ on the other hand, showing results for $m=0.5, \mathcal{K}=10$ exhibits a singularity; indeed, this may be anticipated given the singularity observed at the outer edge (see figure 2), which serves to drive a singularity across the entire boundary layer.

To summarize, it appears that all the $\mathcal{K}=0$ cases fail at either $x=\gamma U_{\mathrm{p} 0} / \beta$ with the formation of the (predicted) stagnation point of the particle velocity on the wall, or at $x_{\mathrm{s}}$ forced by the singularity in the edge conditions.

In the case of $m=0$ and 0.1 , all cases with $\mathcal{K}>0$ could be computed far downstream, while the critical value of $m=m_{\mathrm{s}}$ (as determined from the edge conditions) provides the upper limit for existence of far-downstream solutions. The nature of both types of observed singularity is analysed in $\$ 5$.

Finally, it is worth noting that the breakdowns observed in a number of results in this section are qualitatively different from those found in the case of the wall-jet boundary layer by Duck et al. (2004). One fundamental difference seems to exist between the wall jet and boundary layer results: at the origin of the wall jet, the transverse particle velocity is equal to the outer edge transverse velocity component across the entire layer. This non-zero particle transverse velocity leads to a non-zero value at the wall. The situation with the boundary layer is quite different. The evolution begins with 
zero normal particle velocity on the wall (indeed, this is maintained downstream if $\mathcal{K}=0$ ).

\section{Local analysis of boundary-layer singularities}

We observed in $\$ 3$ that the flow at the edge of the boundary layer may develop a singularity at a particular location downstream of the leading edge. However, as the numerics in $\$ 4$ reveal, a singularity can also develop on the wall that prevents further computation of the boundary-layer solution. In this section, we examine the two types of singularity in more detail; they represent a failure of the dilute model and are not related to any pathological behaviour of $u_{\text {pe }}$ or $\alpha_{\mathrm{e}}$.

$$
\text { (a) The } \mathcal{K}=0 \text { case }
$$

Examination of the equation for $u_{\mathrm{p}},(3.3 a)$, at the wall for $\mathcal{K}=0$, and assuming that $v_{\mathrm{p}}=0$ for all $x$ leads to

$$
U_{\mathrm{pw}}\left(U_{\mathrm{pw}_{x}}+\frac{\beta}{\gamma}\right)=0
$$

where we have written $U_{\mathrm{pw}}$ for $U_{\mathrm{p}}(y=0)$. Consequently, there are two solutions for $u_{\mathrm{p}}$ : (i) zero, and (ii) one with a gradient of $-\beta / \gamma$. Following from (4.1), assuming $U_{\mathrm{p} 0}=1$, we have that

$$
U_{\mathrm{pw}}=1-\frac{\beta x}{\gamma}, \quad \text { for } x<\frac{\gamma}{\beta} .
$$

Under the stated assumption that $v_{\mathrm{p}}=0$ at the surface, the $y$-derivative of the vertical momentum equation gives

$$
U_{\mathrm{pw}} E_{\mathrm{w}}^{\prime}+E_{\mathrm{w}}^{2}+\frac{\beta}{\gamma} E_{\mathrm{w}}=0, \quad E_{\mathrm{w}} \equiv\left(\partial v_{\mathrm{p}} / \mathrm{\partial} y\right)_{y=0},
$$

since $v_{y}$ is always zero on the wall. This equation may be exactly integrated to give

$$
\frac{E_{\mathrm{w}}}{E_{\mathrm{w}}+\frac{\beta}{\gamma}}=C\left(1-\frac{\beta x}{\gamma}\right) \text {. }
$$

The quantity $C$ is simply determined by evaluating $E_{\mathrm{w}}$ at the leading edge, and for all of the wedge flow cases, $E_{\mathrm{w}}(0)=0$, making $C=0$. Therefore,

$$
E_{\mathrm{w}}=0, \quad \text { for } x \geq 0, \quad \text { for } 0<m<1 \text {. }
$$

Evaluating the $\alpha$ equation at the wall shows that $u_{\mathrm{p}} \alpha$ is locally constant, hence

$$
\alpha=\frac{\alpha_{0}}{U_{\mathrm{pw}}}=\frac{\alpha_{0}}{1-\frac{\beta x}{\gamma}}, \quad U_{\mathrm{pw}}>0 .
$$

Therefore, the volume fraction is singular part way along the wall, and so the model breaks down. This breakdown has been noted by other investigators, though Wang \& Glass (1988) continued their computation through the singularity. This is not correct, since no continuation beyond such a singularity is possible without including additional physics into the model equations. It is 
interesting to note that this type of breakdown was not observed in wall jets, the fundamental difference being again that the vertical particle velocity on the wall is zero at the leading edge and remains identically zero downstream.

The appropriate local scaling near $x_{\mathrm{s}}=\gamma / \beta$ is

$$
u_{\mathrm{p}}=\frac{\beta}{\gamma}\left(x_{\mathrm{s}}-x\right) \tilde{U}_{\mathrm{p}}(\tilde{\eta}), \quad v_{\mathrm{p}}=\left(\frac{\beta}{\gamma}\right)^{2}\left(x_{\mathrm{s}}-x\right) \tilde{V}_{\mathrm{p}}(\tilde{\eta}), \quad \alpha=\frac{\tilde{\alpha}(\tilde{\eta})}{x_{\mathrm{s}}-x}, \quad \tilde{\eta}=\frac{\gamma}{\beta} \frac{y}{x_{\mathrm{s}}-x}
$$

Substitution into the particle equations gives

$$
-\tilde{U}_{\mathrm{p}}^{2}+\tilde{\eta} \tilde{U}_{\mathrm{p}} \tilde{U}_{\mathrm{p} \tilde{\eta}}+\tilde{V}_{\mathrm{p}} \tilde{U}_{\mathrm{p} \tilde{\eta}}=k \tilde{\eta}-\tilde{U}_{\mathrm{p}}, \quad \tilde{U}_{\mathrm{p}}\left(\tilde{\eta} \tilde{V}_{\mathrm{p} \tilde{\eta}}-\tilde{V}_{\mathrm{p}}\right)+\tilde{V}_{\mathrm{p}} \tilde{V}_{\mathrm{p} \tilde{\eta}}=-\tilde{V}_{\mathrm{p}},
$$

where $k=u_{y}(x, y=0)$. The particle continuity equation becomes

$$
\tilde{\eta}\left(\tilde{\alpha} \tilde{U}_{\mathrm{p}}\right)_{\tilde{\eta}}+\left(\tilde{\alpha} \tilde{V}_{\mathrm{p}}\right)_{\tilde{\eta}}=0
$$

The behaviour for large $\tilde{\eta}$ is important to the matching process. We find that

$$
\tilde{U}_{\mathrm{p}} \sim c_{1} \tilde{\eta}+c_{3}+1-\frac{k}{c_{1}}, \quad \tilde{V}_{\mathrm{p}} \sim c_{2} \tilde{\eta}+\frac{c_{2}\left(c_{2}+1\right)}{c_{1}}, \quad \tilde{\eta} \rightarrow \infty,
$$

which can be shown to match to the $\eta=\mathcal{O}(1)$ zone.

We have examined the nature of this singularity when the Saffman force, (2.4), is included, since it has been reported elsewhere (Osiptsov 1988) that it removes the 'accumulation' difficulty. For the small-particle asymptotic theory considered in this paper, that is, $\beta \rightarrow \infty$, the Saffman force only slightly modifies the flow up to the immediate neighbourhood of the singularity. The Saffman force does become important near the singularity. Specifically, the above analysis near $x=x_{\mathrm{s}}$ is modified by the replacement of (5.8) with

$$
\tilde{U}_{\mathrm{p}}\left(\tilde{\eta} \tilde{V}_{\mathrm{p} \tilde{\eta}}-\tilde{V}_{\mathrm{p}}\right)+\tilde{V}_{\mathrm{p}} \tilde{V}_{\mathrm{p} \tilde{\eta}}=-\tilde{V}_{\mathrm{p}}-c \frac{\gamma R e^{3 / 4}}{\beta^{3 / 2}} k^{1 / 2}\left(k \tilde{\eta}-\tilde{U}_{\mathrm{p}}\right) .
$$

Thus, for sufficiently small particles, $\beta \geq \mathcal{O}\left(R e^{1 / 2}\right)$, the Saffman force slightly modifies the singularity structure, but does not eliminate the singularity. Obviously, if the particles are not that small, then $\beta$ may not be large enough to satisfy the above criterion, and the Saffman force may well alter the boundary layer well before any accumulation arises.

\section{(b) The $\mathcal{K}>0, m>m_{s}$ case}

When $\mathcal{K}>0$, we have seen that the outer flow develops a singularity for $m>m_{\mathrm{s}}$ (which is independent of $\mathcal{K}$ ). In this regime, it is clear from the results of $\S 4$ that this then triggers a singularity across the entire boundary layer. It would appear (by inspection of both our boundary-layer results and also from (3.9)) that as $x \rightarrow x_{\mathrm{s}}$ (the point of singularity), the boundary-layer solution takes the form

$$
\left.\begin{array}{c}
u=\hat{u}(y)+\cdots, \quad v=\left(x_{\mathrm{s}}-x\right)^{-1 / 2} \hat{v}(y)+\cdots, \quad \alpha=\left(x_{\mathrm{s}}-x\right)^{-1} \hat{\alpha}(y)+\cdots, \\
u_{\mathrm{p}}=\hat{u}_{p}(y)+\cdots, \quad v_{\mathrm{p}}=\left(x_{\mathrm{s}}-x\right)^{-1} \hat{v}_{p}(y)+\cdots .
\end{array}\right\}
$$


Substitution of (5.12) into $(2.5 b),(2.5 c)$ and $(2.5 e)$ leads to the conclusion that $\hat{u}$, $\hat{v}, \hat{u}_{\mathrm{p}}$ and $\hat{\alpha}$ must be constants, together with

$$
\hat{v}_{\mathrm{p}}=-y \hat{u}_{\mathrm{p}}+\text { const. }
$$

Since $\hat{\alpha}$ is a constant, matching with (3.9) necessitates that $\hat{\alpha}=1$, while for the fluid it is clear that

$$
\hat{u}(y)=x_{\mathrm{s}}^{m}, \quad \hat{v}(y)=\text { const. }
$$

Clearly, (5.14) satisfies neither no slip or impermeability, and hence an inner layer is required (indeed the $U_{\eta}(\eta=0)$ distribution in figure $4 b$ is suggestive of this). This layer is defined by $\hat{\eta}=y /\left(x_{\mathrm{s}}-x\right)^{1 / 2}=\mathcal{O}(1)$, in which

$$
u=\hat{U}(\hat{\eta})+\cdots, \quad v=\left(x_{\mathrm{s}}-x\right)^{-1 / 2} \hat{V}(\hat{\eta})+\cdots,
$$

where to leading order, $\alpha, v_{\mathrm{p}}$ and $u_{\mathrm{p}}$ are constant across this layer, and are $\mathcal{O}\left(\left(x_{\mathrm{s}}-x\right)^{-1}\right), \mathcal{O}\left(\left(x_{\mathrm{s}}-x\right)^{-1}\right)$ and $\mathcal{O}(1)$, respectively. The system (determining primarily the fluid motion) is then sufficient to satisfy the necessary conditions on the wall $(y=\hat{\eta}=0)$. Note that this predicts an inverse square-root singularity in the fluid wall-shear distribution $U_{\eta}(\eta=0)$, in line with the singular behaviour as observed in figure $4 b$.

\section{Boundary layers with $\mathcal{K}<0$}

When gravitational forces act away from the wall, an analysis of the leading-edge region, $x \ll 1$, indicates that $v_{\mathrm{p}}>0$ near $y=0$. This raises the possibility of shock formation (see also Foster et al. (2003)). A naive application of the numerical schemes implemented in the previous sections of this work leads, in this $\mathcal{K}<0$ regime, to inconsistent results (i.e. results that are dependent on resolution parameters).

It is clear that for $\mathcal{K}<0$, close to the wall characteristics will be directed outwards from the boundary (since gravitational forcing in the transverse direction will overcome Stokes drag adjacent to an impermeable wall). Therefore, any numerical scheme must apply boundary conditions on $y=0$ when $\mathcal{K}<0$. While there are some similarities in this case with the study of Duck et al. (2004), there is one significant difference, namely $v_{\mathrm{p}}(y=0)>0$ immediately at $x=0$ (in the wall jet problem, this feature was only found to occur at a finite downstream location).

Inspection of the system $(2.5 a)-(2.5 e)$ suggests that a local analysis should take the form

$$
u_{\mathrm{p}}=U_{\mathrm{p} 0}+\cdots, \quad v_{\mathrm{p}}=x \hat{v}_{\mathrm{p}}(\hat{\eta})+\cdots, \quad \alpha=\hat{\alpha}(\hat{\eta})+\cdots,
$$

where $\hat{\eta}=y / x^{2}$ and the effect of the fluid is relatively benign in this region. The equations resulting from (6.1) are then

$$
\hat{v}_{\mathrm{p} \hat{\eta}}\left(\hat{v}_{\mathrm{p}}-2 U_{\mathrm{p} 0} \hat{\eta}\right)+U_{\mathrm{p} 0} \hat{v}_{\mathrm{p}}=-\mathcal{K} \cos \theta, \quad \hat{v}_{\mathrm{p}} \hat{\alpha} \hat{\eta}-\frac{U_{\mathrm{p} 0}}{2} \hat{\eta} \hat{\alpha}_{\hat{\eta}}+\hat{\alpha} \hat{v}_{\mathrm{p} \hat{\eta}}=0 .
$$

Equations (6.2) are reminiscent of those studied in some detail by Duck et al. (2004). Considering the first of (6.2), then as $\hat{\eta} \rightarrow \infty, \hat{v}_{\mathrm{p}} \rightarrow-\mathcal{K} \cos \theta / U_{\mathrm{p} 0}$. It is 

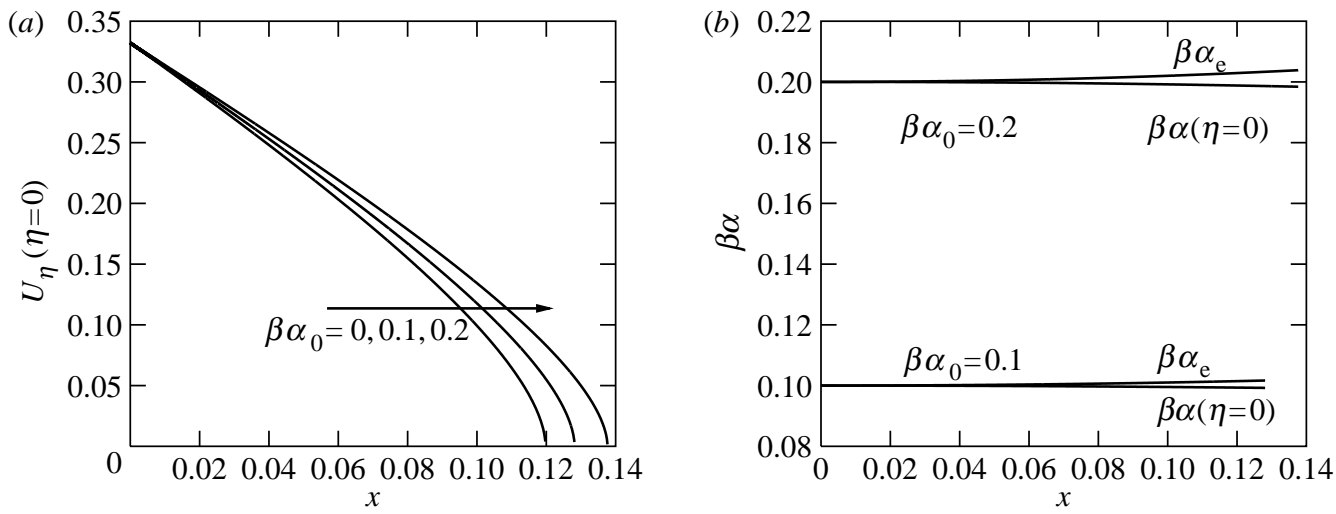

Figure 5. The development of wall and edge values with $x$ for $u_{\mathrm{e}}(x)=1-x$ for $\mathcal{K}=0, \beta / \gamma=1$.

possible to determine an exact solution for $\hat{v}_{\mathrm{p}}$, namely

$$
K_{1}=\frac{\left(\hat{v}_{\mathrm{p}}+\frac{\mathcal{K} \cos \theta}{U_{\mathrm{p} 0}}\right)^{2}}{\hat{v}_{\mathrm{p}}-U_{\mathrm{p} 0} \hat{\eta}+\frac{\mathcal{K} \cos \theta}{2 U_{\mathrm{p} 0}}},
$$

where $K_{1}$ is a constant. The exact solution consistent with the matching condition is $\hat{v}_{\mathrm{p}}=-\mathcal{K} \cos \theta / U_{\mathrm{p} 0}$, corresponding to the constant $K_{1}=0$. This solution is not valid near the wall, however. If we impose a boundary condition at $\hat{\eta}=0$ of $\hat{v}_{\mathrm{p}}=0$, then this leads to a different value of the constant $K_{1}$, namely, $K_{1}=2 \mathcal{K} \cos \theta / U_{\mathrm{p} 0}$, and for this case, the exact solution (6.3) becomes $\hat{v}_{\mathrm{p}}=\sqrt{-2 \mathcal{K} \cos \theta \hat{\eta}}$. These solutions connect at $\hat{\eta}=-\mathcal{K} \cos \theta /\left(2 U_{\mathrm{p} 0}\right)=\hat{\eta}_{\mathrm{s}}$, giving a discontinuity in slope for $\hat{v}_{\mathrm{p}}$ at this location.

Equation (6.2) can now easily be solved in these two zones. For $\hat{\eta}<\hat{\eta}_{\mathrm{s}}$, requiring that the solution be bounded results in $\hat{\alpha} \equiv 0$, i.e. the region is particle free. In $\hat{\eta}>\hat{\eta}_{\mathrm{s}}$, the only solution is $\hat{\alpha}=\alpha_{0}$. Thus, a discontinuity in $\alpha$ lies along the parabola $y=-\mathcal{K} \cos \theta x^{2} /\left(2 U_{\mathrm{p} 0}\right)$. Further evidence of the existence of discontinuities in the $\alpha$ distribution when $\mathcal{K}<0$ is given in appendix A (which considers the far-downstream regime).

\section{Results for the Howarth edge flow}

It is relatively straightforward to extend the class of $u_{\mathrm{e}}(x)$ from the FalknerSkan-type considered previously; here we will restrict our attention to flows of the linearly retarding type $u_{\mathrm{e}}(x)=1-x$, together with $U_{\mathrm{p} 0}=1$. As such, this represents the dusty analogue to the Howarth (1934) problem.

Figure $5 a$ shows the wall shear stress (of the fluid) distribution for the case $\mathcal{K}=0, \gamma / \beta=1$, for $\beta \alpha=0$ (the clean case), together with $\beta \alpha_{0}=0.1,0.2$. Flow reversal, as expected from the classical clean flow, occurs at a downstream location, but, perhaps unexpectedly an increasing concentration of particles does (slightly) defer flow separation (accompanied by the Goldstein (1948) singularity). The corresponding distributions of both $\alpha_{\mathrm{e}}$ and $\alpha(y=0)$ are shown in figure $5 b$; these indicate a relatively benign response of the particles. The 
corresponding distributions of $u_{\mathrm{p}}(y=0)$ are not shown, since these take on the now familiar linear form, namely $u_{\mathrm{p}}(y=0)=1-\beta x / \gamma$ (assuming $\left.0<x<\gamma / \beta\right)$. It is clear that the Stokes drag term in the fluid streamwise momentum equation slightly delays flow separation; on the wall, clearly $u-u_{\mathrm{p}}<0$, and this has an accelerating effect on the flow (although the pressure gradient appears slightly more adverse than in the particle-free case).

\section{Final comments}

In this work, we have shown that, within the confines of the boundary-layer system, the particle-laden dusty-gas model may predict one of three severe events, including two types of singularity (in which the dilute nature of the flow is not preserved) or the formation of shock-like transitions in the particle distribution. Clearly, the singular phenomena are indicative of a failure of the dusty-gas model, at least in the context of a dilute suspension. It is the authors' conjecture that these effects should be 'observable' in comparable laboratorybased experimentation (as areas of significantly increased volume fraction or particle-free zones). Furthermore, we propose that a return to a more broadly applicable set of field equations (whatever they may be) in the vicinity of the breakdown would ameliorate the singular difficulties and allow further downstream progression of the solution. Exactly what additional physical influence is required to be included in order to eliminate the singular structure from the governing equations remains work in progress, including a weakly non-dilute model which may lead to a framework suitable for further investigation.

Clearly, our analysis of the boundary-layer flow makes implicit assumptions regarding the development of an appropriate outer inviscid, irrotational solution to the dusty-gas model. In this work, we have determined the edge conditions applied to the boundary-layer solution as part of the solution procedure. We must recognize, however, that the external flow is in general also non-trivial; this is in contrast to most previous applications of the dusty-gas model in which the external flow field is not exposed to any pressure gradients. The distinction is that, since the pressure gradient only influences the fluid phase and not the particle phase, a general outer solution for which there is no differential motion between the particle and fluid phase is only possible when this pressure forcing is absent. It is, therefore, unsurprising that our analysis demonstrates the presence of an arbitrary constant $U_{\mathrm{p} 0}$ in the leading-edge asymptotics since this constant is associated with the degree of interphase 'slip'.

We conclude by noting that, even though inviscid particle-laden flows have been considered by other authors, and boundary-layer flows have been discussed extensively by the present authors (and others), relatively little work has been done towards a synoptic view of dusty-gas flow phenomena in which the two are both considered formally. As we have noted above, in some circumstances this process is straightforward, for example in the absence of a free-stream pressure gradient, but in general a complete description is rather more challenging.

The authors benefited from a number of discussions on this work with Dr David Harris. Much of this work was performed while the authors were in residence at the Isaac Newton Institute for the Mathematical Sciences, Cambridge. The referees of the paper also provided a number of useful comments. 


\section{Appendix A. Large- $x$ structure}

Although for a wide choice of parameter values, the boundary-layer flow appears to suffer a breakdown of one kind or another, it is possible to develop asymptotic solutions, valid in the far downstream limit, which here we assume do exist.

$$
\text { (a) } \mathcal{K}=0 \text { case }
$$

In general, for large $x$ and for $\mathcal{K}=0$, we might expect the familiar self-similar form for the fluid, and thus we define the usual similarity variable $\eta=y x^{(m-1) / 2}$. Then, the large- $x$ expansions are a generalization of those found in Wang \& Glass (1988),

$\left(u, u_{\mathrm{p}}\right) \sim x^{m}\left(U_{0}(\eta), U_{0}(\eta)\right)+\cdots,\left(v, v_{\mathrm{p}}\right) \sim x^{(m-1) / 2}\left(V_{0}(\eta), V_{0}(\eta)\right)+\cdots, \alpha=\alpha_{\infty}+\cdots$,

(it is straightforward to show that to leading order $\alpha$ takes on its freestream value $\alpha_{\infty}$ across the entire layer). The fluid continuity equation is merely

$$
m U_{0}+\frac{m-1}{2} \eta U_{0}^{\prime}+V_{0}^{\prime}=0
$$

while a combination of the two streamwise momentum equations leads to the classical Falkner-Skan equation, but with a modified 'viscosity',

$$
\left(1+\alpha_{\infty}\right)\left(m U_{0}^{2}+\frac{m-1}{2} \eta U_{0} U_{0}^{\prime}+V_{0} U_{0}^{\prime}-m\right)=U_{0}^{\prime \prime},
$$

subject to $U_{0}(0)=V_{0}(0)=0, U_{0}(\eta \rightarrow \infty) \rightarrow 1$.

\section{(b) $\mathcal{K}>0$ : a constant-thickness layer}

In this case, it turns out that the large- $x$ structure of the boundary layer no longer exhibits the Falkner-Skan-type similarity in the presence of sedimentation. This is because the vertical velocity of the fluid decays downstream as $x^{(m-1) / 2}$, whereas the Stokes settling speed does not decay and this will dominate the structure sufficiently far downstream. As a consequence, the large- $x$ behaviour is given by

$$
\begin{aligned}
& u=x^{m} U_{0}(Y)+\cdots, \quad u_{\mathrm{p}}=x^{m} U_{\mathrm{p} 0}(Y), \quad \alpha=\alpha_{\infty}+\cdots, \\
& v=\left(\alpha_{\infty} \beta\right)^{-1 / 2}\left(x^{m-1} V_{0}(Y)+\cdots\right), \quad v_{\mathrm{p}}=\left(\alpha_{\infty} \beta\right)^{-1 / 2}\left(\frac{\beta}{\gamma} V_{\mathrm{p} 0}(Y)+\cdots\right),
\end{aligned}
$$

where it is convenient to choose a scaled variable, $y=Y / \sqrt{\alpha_{\infty} \beta}$, and we have utilized the condition that to leading order $\alpha=\alpha_{\infty}$ (the far downstream, freestream value). The parameter that naturally occurs in this analysis is the solution to the problem for $V_{\mathrm{p} 0}$ is easily evaluated, and is given by $V_{\mathrm{p} 0}=-\mathcal{K}^{\prime}$, where $\mathcal{K}^{\prime} \equiv \mathcal{K}(\gamma / \beta)^{2}\left(\alpha_{\infty} \beta\right)^{1 / 2} \cos \theta$. With this result, solutions for $U_{0}, U_{\mathrm{p} 0}, V_{0}, \ldots$ 
are found to be

$$
\left.\begin{array}{l}
U_{0}=1-\mathrm{e}^{-\lambda Y}, \quad \text { where } \lambda=\frac{\sqrt{1+4 \mathcal{K}^{\prime 2}}-1}{2 \mathcal{K}^{\prime}}, \\
=\left(\lambda^{2}-1\right) \mathrm{e}^{-\lambda Y}+1, \quad V_{0}=-m Y+\frac{m}{\lambda}\left(1-\mathrm{e}^{-\lambda Y}\right) .
\end{array}\right\}
$$

Note that particles slip at the surface, and accumulate there under gravity, that is,

$$
\left.U_{\mathrm{p} 0}\right|_{Y=0}=\lambda^{2},\left.\quad V_{\mathrm{p} 0}\right|_{Y=0}=-\mathcal{K}^{\prime} .
$$

These asymptotic wall values can clearly be seen in the results shown in figure $3 b$.

It turns out that the volume fraction for this case is double-structured, with a portion of it decaying to edge values over a much larger scale-namely $y \sim x^{1-m}$; details are not included here for brevity.

\section{(c) $\mathcal{K}<0$ : a shock transition in particle distribution}

In this case, there is an important distinction with the $\mathcal{K}>0$ regime; the $\hat{\eta}=y / x^{1-m}$ region takes on some significance in these flows. Considering an $\hat{\eta}=\mathcal{O}(1)$ zone, the solution develops as follows:

$$
\left(u, u_{\mathrm{p}}\right)=x^{m}\left(\hat{U}_{0}(\hat{\eta}), \hat{U}_{\mathrm{p} 0}(\hat{\eta})\right)+\cdots, \quad\left(v, v_{\mathrm{p}}\right)=\left(\hat{V}_{0}(\hat{\eta}), \hat{V}_{\mathrm{p} 0}(\hat{\eta})\right)+\cdots, \alpha=\hat{A}_{0}(\hat{\eta})+\cdots
$$

It is immediately apparent that the Stokes drag term dominates the leading order, yielding

$$
\hat{U}_{\mathrm{p} 0}=\hat{U}_{0}, \quad \hat{V}_{\mathrm{p} 0}=\hat{V}_{0}-\frac{\gamma \mathcal{K}^{*}}{\beta},
$$

where $\mathcal{K}^{*} \equiv \mathcal{K} \cos \theta$. At leading order, the particle continuity equation reduces to

$$
\left[\hat{U}_{0}(m-1) \hat{\eta}+\hat{V}_{0}-\frac{\gamma \mathcal{K}^{*}}{\beta}\right] \hat{A}_{0}^{\prime}=0
$$

from which we may infer that $\hat{A}_{0}=$ const., provided that the multiplying term in brackets does not possess a zero (we say more regarding this below). Here the prime notation indicates differentiation with respect to $\hat{\eta}$.

At next order in the (fluid/particle-phase) streamwise momentum equations, we can eliminate the interphase drag term $\hat{U}_{1}-\hat{U}_{\mathrm{p} 1}$ to obtain

$$
\left(1+\gamma \hat{A}_{0}\right)\left[\hat{V}_{0} \hat{U}_{0}^{\prime}-\hat{U}_{0} \hat{V}_{0}^{\prime}\right]-\frac{\gamma^{2} \mathcal{K}^{*}}{\beta} \hat{A}_{0} \hat{U}_{0}^{\prime}=m\left(1+\gamma \alpha_{\infty}\right)
$$

where $\alpha_{\infty}=\hat{A}_{0}(\infty)$ is the far downstream, freestream value of $\alpha$.

Finally, the continuity equation for the fluid can be written as

$$
\hat{V}_{0}^{\prime}+(m-1) \hat{\eta} \hat{U}_{0}^{\prime}+m \hat{U}_{0}=0 .
$$

At the edge of the layer, we anticipate a solution to (A 9) in which the particles are advected with the fluid, but with an additional gravitational settling 
component. Indeed, such a solution is readily found:

$$
\hat{U}_{0}=1, \quad \hat{A}_{0}=\alpha_{\infty}, \quad \hat{V}_{0}=-m \hat{\eta}+\delta^{*},
$$

where $\delta^{*}$ is a displacement thickness term. We note that $\delta^{*}$ is a parameter that represents 'history' effects in that it depends upon the evolution of the boundary layer from the leading edge. Furthermore, given (A 11), we may determine the particle flow field from (A 7). The simple outer solution (A 11) must either be matched to an inner region adjacent to the boundary, or fail at a critical point. In fact, it is the latter that occurs here, since if we define

$$
\phi(\hat{\eta})=(m-1) \hat{\eta} \hat{U}_{0}+\hat{V}_{0},
$$

then we can see that there is potential for a critical location to exist at $\hat{\eta}=\hat{\eta}_{\mathrm{s}}=\delta^{*}-\gamma \mathcal{K}^{*} / \beta$, where $\phi\left(\hat{\eta}_{s}\right)=\gamma \mathcal{K}^{*} / \beta$ from (A 8). We, therefore, continue our analysis with the aim of developing a solution with a shock-like transition in $\hat{A}_{0}(\hat{\eta})$ at a location $\hat{\eta}=\hat{\eta}_{\mathrm{s}}$, at which point $\phi$ remains continuous, but is nondifferentiable.

The quantity $\phi$ is a convenient change of variable and we may rewrite (A 9) (after using the fluid continuity equation) as,

$$
\left(1+\gamma \hat{A}_{0}\right)\left[m\left(\phi^{\prime}\right)^{2}-\phi \phi^{\prime \prime}\right]+\frac{\gamma^{2} \mathcal{K}^{*}}{\beta} \hat{A}_{0} \phi^{\prime \prime}=m\left(1+\gamma \alpha_{\infty}\right) .
$$

The solution for $\phi$ below the critical shock location $\hat{\eta}_{\mathrm{s}}$ is clearly dependent on the conditions applied at the boundary for the particle concentration $\alpha$. However, we know that $A_{0}$ must be constant in this layer, but equally we expect that gravity will move particles away from the wall. If particles are not replenished from the wall (i.e. if there is no source of particles at the wall), then the obvious conclusion is that a particle-free region must develop with $\hat{A}_{0}=0$; indeed, the near-leading-edge region, studied in $\$ 6$ strongly supports this concept. This, therefore, leads us to consider solutions to (A 13) with $\hat{A}_{0}=0$, for which we must solve

$$
m \phi^{\prime 2}-\phi \phi^{\prime \prime}=m\left(1+\gamma \alpha_{\infty}\right),
$$

which can be integrated to give

$$
\frac{\mathrm{d} \phi}{\mathrm{d} \hat{\eta}}=-\left(1+\gamma \alpha_{\infty}\right)^{1 / 2}\left(1-c_{1} \phi^{2 m}\right)^{1 / 2},
$$

where $c_{1}$ is an arbitrary constant. Therefore, if one can match the solution in $\hat{\eta}>\hat{\eta}_{\mathrm{s}}$ given by (A 11) to the solution (A 15) at $\hat{\eta}_{s}$, then this will define a consistent shock-like transition in the particle distribution. The physical mechanism of this discontinuous distribution is a simple balance of drag due to entrainment of the fluid into the boundary layer in competition with gravitational forcing - the two influences balancing at $\hat{\eta}_{\mathrm{s}}$.

To match across the shock at $\hat{\eta}_{\mathrm{s}}$ we first note that (A 11) yields $\phi=\delta^{*}-\hat{\eta}$ for $\hat{\eta}>\hat{\eta}_{\mathrm{s}}$. Secondly, we see that

$$
\hat{\eta}=-\int_{0}^{\phi} \frac{1}{1+\gamma \alpha_{\infty}}\left(1+c_{1} \chi^{2 m}\right)^{-1 / 2} \mathrm{~d} \chi
$$


for $\hat{\eta}<\hat{\eta}_{\mathrm{s}}$. Finally, since $\phi\left(\hat{\eta}_{\mathrm{s}}\right)=\gamma \mathcal{K}^{*} / \beta$, we can determine $c_{1}$ implicitly through the integral condition

$$
\delta^{*}-\frac{\gamma \mathcal{K}^{*}}{\beta}=\frac{-1}{1+\gamma \alpha_{\infty}} \int_{0}^{\gamma \mathcal{K}^{*} / \beta}\left(1+c_{1} \chi^{2 m}\right)^{-1 / 2} \mathrm{~d} \chi
$$

As an aside, we note that when $m=0$ there is some degeneracy and the coefficient $c_{1}$ is readily determined to be

$$
c_{1}=1-\left(\frac{\gamma \mathcal{K}^{*}}{\delta^{*} \beta-\gamma \mathcal{K}^{*}}\right)^{2} \frac{1}{1+\gamma \alpha_{\infty}} .
$$

Since $\phi^{\prime}=-\hat{U}_{0}$, we can see that (for $m \neq 0$ ), the resulting value of $\hat{U}_{0}(\hat{\eta}=0)$ is given by

$$
\hat{U}_{0}(\hat{\eta}=0)=\left(1+\gamma \alpha_{\infty}\right)^{1 / 2},
$$

which will not satisfy no-slip (although $\hat{V}(\hat{\eta}=0)=0$ ). To reduce the fluid velocity to zero at the boundary, we require an inner layer spanned by $\eta=$ $y / x^{(1-m) / 2}$ (again), in which the scalings are

$$
u=x^{m} U_{0}^{*}(\eta)+\cdots, \quad v=x^{(m-1) / 2} V_{0}^{*}(\eta)+\cdots,
$$

while $v_{\mathrm{p}}=-\gamma \mathcal{K}^{*} / \beta$ and $u_{\mathrm{p}}=x^{m} \hat{U}_{0}(\hat{\eta}=0)$ are both constant across this layer ( $\alpha$ is, of course, zero).

The equations determining the fluid motion in this inner region are of the Falkner-Skan type, i.e.

$$
\begin{gathered}
m U_{0}^{*}-\frac{1-m}{2} \eta U_{0 \eta}^{*}+V_{0 \eta}^{*}=0, \\
U_{0}^{*}\left(m U_{0}^{*}-\frac{1-m}{2} \eta U_{0 \eta}^{*}\right)+V_{0}^{*} U_{0 \eta}^{*}=U_{0 \eta \eta}^{*}+m\left(1+\alpha_{\infty} \beta\right),
\end{gathered}
$$

the requirement being that $U_{0}^{*}(\eta \rightarrow \infty)$ matches to (A 19) (or unity when $m=0$ ), together with $U_{0}^{*}(\eta=0)=V_{0}^{*}(\eta=0)=0$. Given the particle-free nature of this zone, it is perhaps hardly surprising that the flow takes on a form closely related to the classical (clean) form. Solution of the inner flow then closes the problem. Indeed, the Falkner-Skan-type behaviour in the inner region can be anticipated since the flow is essentially a clean (that is, particle-free) problem there. We conclude by noting that this analysis implicitly assumes that $m<1 / 2$; given the breakdown/singularity witnessed earlier in the paper, if $m>m_{\mathrm{s}}<1 / 2$, this is unlikely to be a significant restriction.

\section{References}

Asmolov, E. S. 1995 Dusty gas flow in a laminar boundary layer over a blunt body. J. Fluid Mech. 305, 29.

Duck, P. W., Hewitt, R. E. \& Foster, M. R. 2004 On the spatial development of a dusty wall jet. J. Fluid Mech. 514, 385. (doi:10.1017/S0022112004000205)

Féraille, T. \& Casalis, G. 2003 Channel flow induced by wall injection of fluid and particles. Phys. Fluids 15, 348. (doi:10.1063/1.1530158) 
Foster, M. R., Duck, P. W. \& Hewitt, R. E. 2003 The unsteady Kármán problem for a dilute suspension. J. Fluid Mech. 474, 379. (doi:10.1017/S0022112002002690)

Glauert, M. B. 1956 The wall jet. J. Fluid Mech. 1, 625.

Goldstein, S. 1948 On laminar boundary-layer flow near a position of separation. Q. J. Mech. Appl. Math. 1, 43.

Hernández, J. A. 2001 Instabilities induced by concentration gradients in dusty gases. J. Fluid Mech. 435, 247. (doi:10.1017/S0022112001003858)

Howarth, L. 1934 On calculation of the steady flow in the boundary layer near the surface of a cylinder in a stream. Rep. Memor. Aero. Res. Coun. Lond. No. 1632.

Marble, F. E. 1970 Dynamics of dusty gases. Ann. Rev. Fluid Mech. 2, 397. (doi:10.1146/annurev. fl.02.010170.002145)

Narayanan, C. \& Lakehal, D. 2002 Temporal instabilities of a mixing layer with uniform and nonuniform particle loadings. Phys. Fluids 14, 3775. (doi:10.1063/1.1508771)

Osiptsov, A. N. 1980 Structure of a dispersed-mixture laminar boundary layer on a flat plate. Izv. Akad. Nauk. SSSR Mekh. Zhidk. Gaza. n4, 48.

Osiptsov, A. N. 1988 Dusty gas motion in the initial section of a plane channel or circular pipe. Izv. Akad. Nauk. SSSR Mekh. Zhidk. Gaza. n6, 80.

Osiptsov, A. N. 1997 Mathematical modeling of dusty-gas boundary layers. Appl. Mech. Rev. 50, 357.

Saffman, P. G. 1965 The lift of a small sphere in a slow shear flow. J. Fluid Mech. 22, 385. (See also Corrigendum, 1968, 31, 624.)

Singleton, R. E. 1965 Compressible gas-solid particle flow over a semi-infinite flat plate. Z. Angew. Math. Phys. 16, 421. (doi:10.1007/BF01593921)

Soo, S. L. 1968 Non-equilibrium fluid dynamics-laminar flow over a flat plate. Z. Angew. Math. Phys. 19, 545. (doi:10.1007/BF01594963)

Slater, A. S. \& Young, J. 2001 The calculation of inertial particle transport in dilute gas-particle flows. Int. J. Multiphase Flow 27, 61. (doi:10.1016/S0301-9322(99)00122-6)

Ungarish, M. 1993 Hydrodynamics of suspensions. Berlin: Springer.

Ungarish, M. \& Greenspan, H. P. 1983 On two-phase flow in a rotating boundary layer. Stud. Appl. Math. 69, 145.

Wang, B. Y. \& Glass, I. I. 1988 Compressible laminar boundary-layer flows of a dusty gas over a semi-infinite flat plate. J. Fluid Mech. 186, 223. 